\title{
New Mode Transition of H-bridge Bi-directional DC-DC Converter in Distributed Generation System
}

\author{
A. Yi Lingzhi ${ }^{1,2}$, B. Chen $\mathrm{Yu}^{1,2}$, C. Ren Xuliang ${ }^{1,2}$ and D. Zhang Hao ${ }^{3}$ \\ ${ }^{1}$ Key Laboratory of Intelligent Computing \& Information Processing (Xiangtan University) \\ Hunan Province, Xiangtan, China \\ e-mail: A.450337856@qq.com, B.1538807573@qq.com \\ ${ }^{2}$ Wind power equipment and power conversion 2011 Collaborative Innovation Center \\ Hunan Province Xiangtan,China \\ ${ }^{3}$ CSR Zhuzhou Electric Motor CO., LTD \\ Hunan Province, Zhuzhou, China
}

\begin{abstract}
There are many problems in H-bridge bidirectional DC-DC converter, such as slow dynamic response and bus-bar voltage overshoot etc. The proposed method of dual carrier modulation which can greatly simplify the control method, is used to smooth mode transition. Then a Fractional Order PID (FOPID) controller which can provide an excellent dynamic performance, more flexibility and robustness for the system, is investigated. Finally, simulation model has been built, and an $1 \mathrm{~kW}$ experimental prototype has been built.
\end{abstract}

Keywords: Bi-directional DC-DC converter; mode transition; dual carrier modulation; Fractional Order PID;

\section{INTRODUCTION}

As energy crisis and environmental pollution become more and more serious, the demand of new energy sources is increasingly growing. However, the amount of energy generated is uncontrollable, energy storage source is needed to deal with distributed power source. As shown in Fig.1, the bi-directional converter is a key component in shifting the load.

Various types of bi-directional converters have been reported [1]-[5]. In particular, seamless dynamic model [6] has been proposed and the bi- directional DC-DC converter is much easier to be analyzed and controlled.

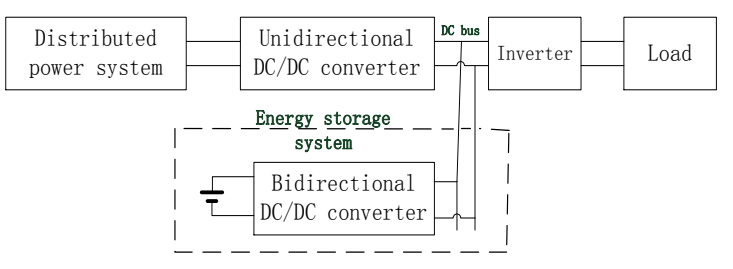

Fig.1 Energy storage block of distributed power source

The working mode of this converter [7] is judged by detecting the DC bus voltage. For it is easy to be interfered, there are false positives caused by false switch. The mode transition of converter [8] is judged by polarity of bus voltage loop. It can restrain the high frequency component, reduce wrong judgement and decrease bus voltage overshoot as well as enhance the dynamic response of the mode transition, but there are only two working modes. In distributed power source, the converter efficiency can be improved by increasing the battery voltage range up to around the DC-link bus line. A lower peak current can be obtained and the transmission loss in the wire can be reduced. Therefore, the operating voltage is partially overlapped, and the converter needs to be operated within four modes, including Buck charging, Buck discharge, Boost charging and Boost discharge. The topology of converter is shown in Fig. 2. 


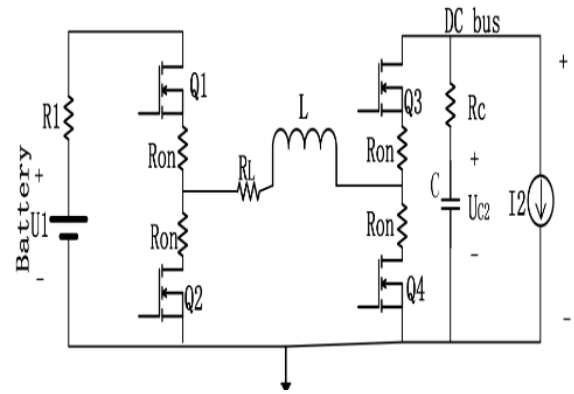

Fig. 2 H-bridge bi-directional DC-DC converter

Due to the simplicity in design and good performance, PID controllers belong to the dominating industrial controllers and hence there has been a continuous attempt to improve their quality and robustness [9]. One of the ways to improve the traditional PID controllers is to use fractional order controllers. In [10], a PSO-based FOPID controller is designed as an automatic voltage regulator (AVR) system to show that the proposed controller is more robust than the classical integer-order PID in presence of uncertainties in generator and exciter model. In [11], [12] FOPID controller is designed to demonstrate its superiority in time domain performance of AVR system with uncertainties in the system components such as generator gain and time constant, exciter, and amplifier. Due to its flatness in the phase margin contribution with wider bandwidth than integer-order PID, FOPID controller surpasses integer-order PID. Therefore, better damping and lower overshoot can be obtained by using FOPID.

In [13], designing of fractional order PID controller for Boost converter can provide much better start-up response and have a good dynamic response. This paper presents a control scheme based in a double control loop, where the outer voltage loop of the bi-directional DC-DC converter is controlled by using fractional order PID to improve the robustness and the dynamic response of the mode transition.

This paper is organized as follows. Section II presents the method of dual carrier modulation. Section III reviews fractional order PID controller and digital implementation. Section VI includes some simulation and experimental results and finally Section VII concludes the paper.

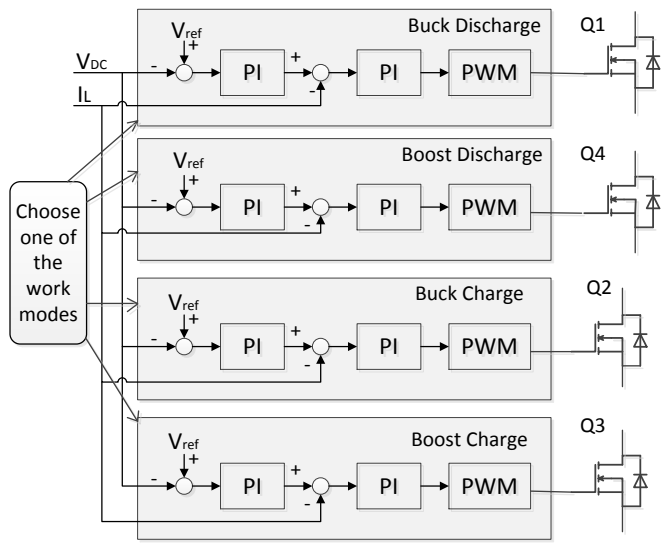

Fig.3-a Control block diagram of traditional H-bridge

bi-directional DC - DC converter

\section{THE PROPOSED METHOD OF DUAL CARRIER MODULATION}

The control block of traditional H-bridge bidirectional DC-DC converter is shown in Fig. 3-a. ( $\mathrm{U}_{\mathrm{DC}}$ for DC bus voltage; $\mathrm{I}_{\mathrm{L}}$ for inductance current; $\mathrm{U}_{\text {ref }}$ for reference voltage). The drive mode and switch state is listed in table 1-a. The work mode can be selected according to the voltage and current polarity. If a PI controller is used, the required convergence time would prevent it from quick response during mode transition. Moreover, the overall mode-select and duty-cycle control process would be very complicated.

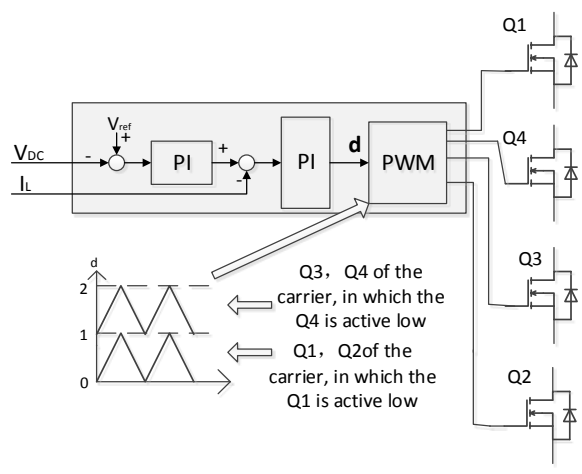

Fig.3-b Control block diagram of dual carrier

To get fast and smooth automatic mode transition, the introduction of dual carrier modulation is shown in Fig.3-b. In H-bridge circuit topology, Q1, Q2, Q3, Q4 were used respectively for complementary symmetry driving signals. Switch state is listed in table 1-b (1 is on, 0 is off, $\sim \mathrm{PWM}$ is complement with PWM). The 
integration of the above four control modes can be simplified into two control modes.

Tab.1-a

\begin{tabular}{|c|c|c|c|c|}
\hline Switch & Q1 & Q2 & Q3 & Q4 \\
\hline Boost Discharge & 1 & 0 & 0 & PWM \\
\hline Buck Charge & 0 & 0 & PWM & 0 \\
\hline Buck Discharge & PWM & 0 & 0 & 0 \\
\hline Boost charge & 0 & PWM & 1 & 0 \\
\hline
\end{tabular}

Tab.1-b

\begin{tabular}{|l|c|c|c|c|}
\hline Switch & Q1 & Q2 & Q3 & Q4 \\
\hline $\begin{array}{c}\text { Boost Discharge } \\
\text { Buck Charge }\end{array}$ & 1 & 0 & $\sim P W M$ & PWM \\
\hline $\begin{array}{c}\text { Buck Discharge } \\
\text { Boost Charge }\end{array}$ & PWM & $\sim$ PWM & 1 & 0 \\
\hline
\end{tabular}

In mode transition, the duty ratio $\mathrm{d}$ is discontinuous, seen in Fig.4-a, where the duty ratio of $\mathrm{Q} 1$ is $0<\mathrm{d}<1$. If $\mathrm{d} 2$ is raised up to 1 , a continuous duty cycle can be got, seen in Fig.4-b. The duty cycle $\mathrm{d}$ can be divided into two stage. One is varied from 0 to 1 , while the other is varied from 1 to 2 . When $0<\mathrm{d}<1, \mathrm{Q} 1$ is driven by the duty cycle d in PWM, and Q4 is off. When $1<\mathrm{d}<2$, Q1 is on, Q4 is driven by the duty cycle d-1 in PWM. The same to Q2 and Q3.

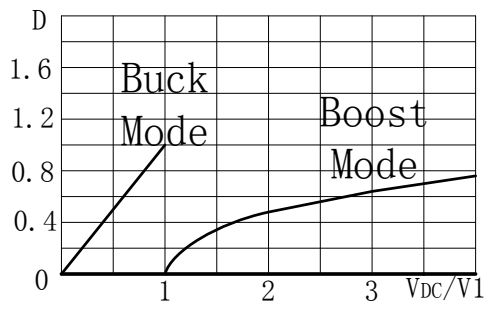

Fig.4-a Control of Discontinuous

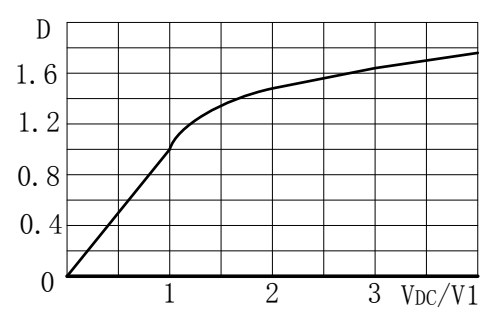

Fig.4-b Control of Continuous

\section{THE FOPID CONTROLLER}

\section{A. Fractional calculus}

Fractional calculus is a branch of mathematical analysis that studies the possibility of taking real number power of the differential operator and integration operator. There are several ways to define fractional order derivatives and integrals. Among all the different definitions, the definition which has been proposed by Grünwald-Letnikov is the most usual one [14]. The definition is as follows:

$$
\begin{aligned}
& { }_{a} D_{t}^{r} f(t)=\lim _{h \rightarrow 0} h^{-r} \sum_{j=0}^{\left[\frac{t-a}{h}\right]}(-1)^{j}\left(\begin{array}{l}
r \\
j
\end{array}\right) f(t-j h)_{(1)} \\
& =\lim _{h \rightarrow 0} h^{-r} \Delta_{h}^{r} f(t)
\end{aligned}
$$

Where [.] mean the integer part, $h$ is the sampling time for calculating the step size and $\Delta_{h}^{r} f(t)$ is the generalized finite difference of order $r$ with step length $h$

The Laplace transforms of formula (1), of signal $f(t)$ at $\mathrm{t}=0$ for order $\mathrm{r}$ is given by

$$
L\left[{ }_{a} D_{t}^{r} f(t)\right]=s^{r} L[f(t)]=s^{r} F(s)
$$

B. The FOPID Controller and Its Digital Implementation.

By using Laplace transformation, the fractional order PID controller is written as:

$$
G_{c}(s)=K_{P}+K_{\mathrm{I}} s^{-\lambda}+K_{D} s^{\delta},(\lambda, \delta>0)
$$

In order to realize the digital procedure of fractional order PID controller, we need to forget a large number of past time series, and can only consider the recent period of time series [15]. The formula (1) can be approximated as follows:

$$
\begin{aligned}
& { }_{a} D_{t}^{r} f(t) \approx{ }_{t-L} D_{t}^{r} f(t) \\
& \approx h^{-r} \sum_{j=0}^{N(t)} b_{j} f(t-j h)
\end{aligned}
$$

Where $\mathrm{L}$ is the memory Length $(\mathrm{t}>\mathrm{L})$, $N(t)=\min \left\{\left[\frac{t}{h}\right],\left[\frac{L}{h}\right]\right\}$, the binomial coefficient $b_{j}$ can be calculated by the formula (5). 
$b_{0}=1, b_{j}=\left(1-\frac{1+r}{j}\right) * b_{j-1}, j \geq 1$

By setting sample times equivalent to calculation step, the discrete expression of the formula (1) can be got.

$$
Z\left\{D^{r} f(t)\right\} \approx\left(T^{-r} \sum_{j=0}^{\left[\frac{\mathrm{L}}{T}\right]} b_{j} z^{-j}\right) F(z)
$$

By using the well-known Tustin operator, we can directly get the approximation of the discrete fractional calculus equation.

$$
Z\left\{D^{r} f(t)\right\} \approx\left(\frac{2}{T} \frac{1-z^{-1}}{1+z^{-1}}\right) F(z)
$$

Any discrete function $G(z)$ can be approximated by a continue-fraction expansion (CFE), which can be expressed as:

$$
G(z)=a_{0}(z)+\frac{b_{1}(z)}{a_{1}(z)+\frac{b_{2}(z)}{a_{2}(z)+\frac{b_{3}(z)}{a_{3}(z)+\cdots}}}
$$

Where $a_{k}(z)$ and $b_{k}(z)$ are either rational function of variable $\mathbf{Z}$ or constants.

Based on the Combination of Tustin operator, the formula (7) can be expressed as:

$$
\begin{aligned}
& Z\left\{D^{r} f(t)\right\} C F E\left\{\left(\frac{1-z^{-1}}{1+z^{-1}}\right)^{r}\right\} f(z) \\
& =\left(\frac{2}{T}\right)^{r} \frac{P_{p}\left(z^{-1}\right)}{Q_{q}\left(z^{-1}\right)} X(z)
\end{aligned}
$$

Wher $P$ and $Q$ are relatively prime polynomial, $p_{\text {and }} q$ are the order of $P$ and $Q$, respectively.

Based on the Tustin+CFE method, we can get the approximation formula of $z\left\{s^{-0.75}\right\}$ and $z\left\{s^{0.5}\right\}$, by setting $\mathrm{T}=0.001$ and $p=q=4$.

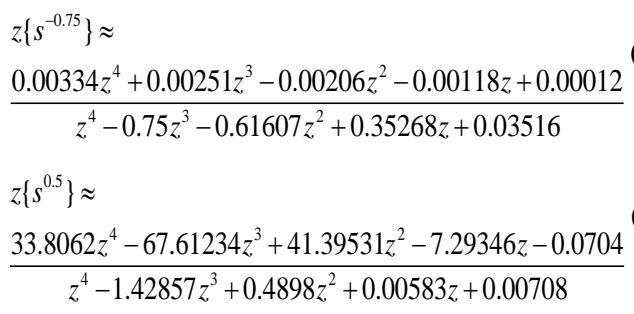

\section{SIMULATION AND EXPERIMENT}

\section{A. The simulation}

Based on Simulink, we developed a simulation program. And the simulation parameters are derived from the photovoltaic energy system, which are listed in table 4 . Table 5 shows the parameters of PI controller and FOPID controller. The simulation waveforms are shown from Fig5a to Fig.5-f (the upper is voltage and the below is inductor current; the positive current is charge and the negative current is discharge).

\begin{tabular}{cc} 
Tab.4 parameters of the Converter \\
\hline Battery voltage $\mathbf{U} 1$ & $\mathbf{3 0 0 V}-\mathbf{5 4 0 V}$ \\
\hline DC bus voltage & $\mathbf{4 0 0 V} \pm \mathbf{2 \%}$ \\
\hline Inductor $\mathbf{L}$ & $\mathbf{8 6 0 u H}$ \\
\hline Capacitor $\mathbf{C}_{\mathbf{0}}$ & $\mathbf{9 6 0 u F}$ \\
\hline Rated Power & $\mathbf{1 0 0 0 W}$ \\
\hline Switching frequency & $\mathbf{2 0 k H z}$ \\
\hline \\
Tab.5 parameters of the of PI controller and FOPID
\end{tabular}

controller

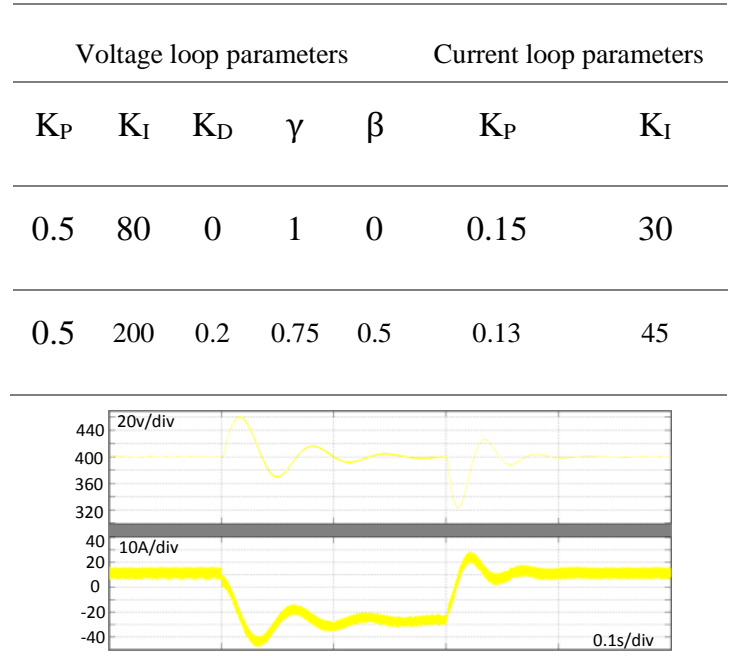

Fig.5-a The simulation waveform of Buck charge/discharge

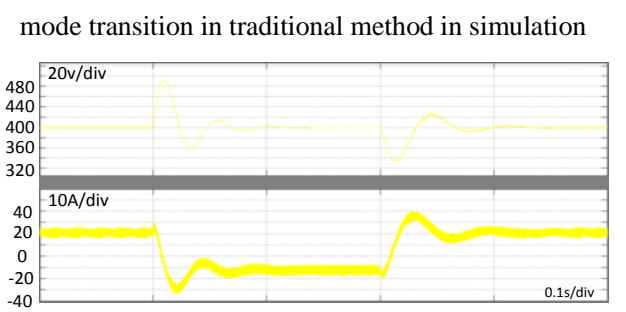

Fig.5-b The simulation waveform of Boost charge/discharge mode transition in traditional method 
In traditional method, see Fig.3-a, the maximum voltage overshoot reaches $80 \mathrm{~V}$ in Buck and the regulation time is about $100 \mathrm{~ms}$; The maximum voltage overshoot reaches $100 \mathrm{~V}$ in Boost and the regulation time is about $120 \mathrm{~ms}$. The simulation waveform is shown in Fig.5-a and Fig.5-b.

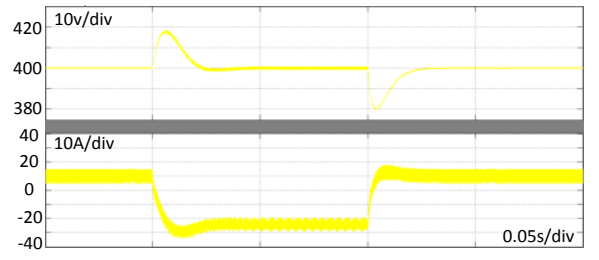

Fig.5-c The simulation waveform of Buck charge/discharge mode transition using PI controller with dual carrier modulation

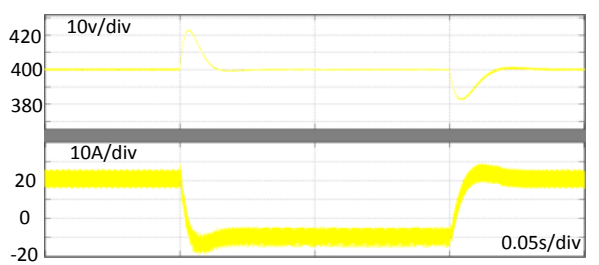

Fig.5-d The simulation waveform of Boost charge/discharge mode transition using PI controller with dual carrier modulation

In Fig.3-b, by using PI controller with dual carrier modulation, the maximum voltage overshoot is less than $20 \mathrm{~V}$ in Buck and the regulation time is about $20 \mathrm{~ms}$; the maximum voltage overshoot is less than $25 \mathrm{~V}$ in Boost, and the regulation time is about $25 \mathrm{~ms}$. The simulation waveform is shown in Fig.5-c and Fig.5-d.

In Fig.3-b, by using FOPID controller in voltage outer loop, the maximum voltage overshoot is less than $5 \mathrm{~V}$ in Buck and the regulation time is about $5 \mathrm{~ms}$; the maximum voltage overshoot is less than $5 \mathrm{~V}$ in Boost, and the regulation time is less than $5 \mathrm{~ms}$. But the steady state error is bigger than PI controller and the current overshoot is bigger in buck, which can meet the output and current requirements without affecting the performance of the converter. The simulation waveform is shown in Fig.5-e and Fig.5-f.

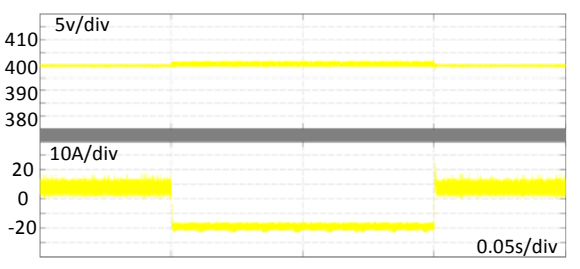

Fig.5-e The simulation waveform of Buck charge/discharge mode transition using FOPID controller with dual carrier modulation

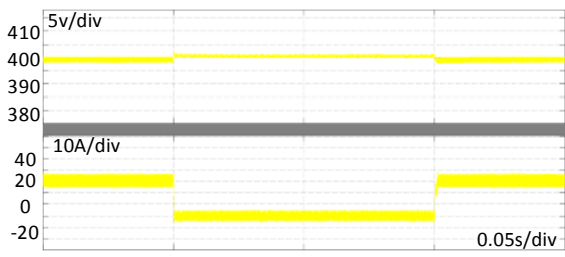

Fig.5-f The simulation waveform of Boost charge/discharge mode transition using FOPID controller with dual carrier modulation

\section{B. Experimental verification}

The experimental prototype consists of controller and power converter. The TMS320F2812 is adopted as the main controller and the power converter is made up of MOSFET IPA60R165CP. A $1 \mathrm{kw}$ experimental prototype is designed, in which the output voltage and the current are $400 \mathrm{~V}$ and $2.5 \mathrm{~A}$ respectively. Using PI controller with dual carrier modulation, the measured voltage and current waveforms are shown in Fig.6-a and Fig.6-d. And using FOPID controller with dual carrier modulation, the measured voltage and current waveforms are shown in Fig.6-c and Fig.6-d

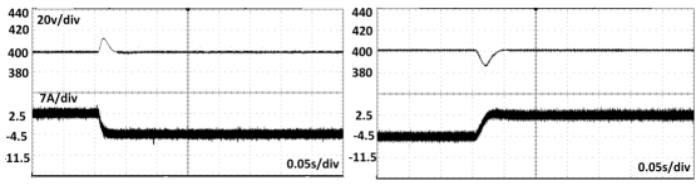

Fig.6-a the experiment waveform of Buck charge/discharge mode transition using PI controller

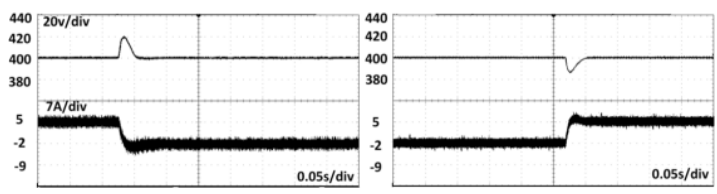

Fig.6-b the experiment waveform of Boost charge/discharge mode transition using PI controller

By using PI controller, the maximum voltage overshoot of mode transition is about $18 \mathrm{~V}$ in buck 
and the regulation time is about $25 \mathrm{~ms}$. The maximum voltage overshoot is about $25 \mathrm{~V}$ in Boost and the regulation time is about $30 \mathrm{~ms}$.

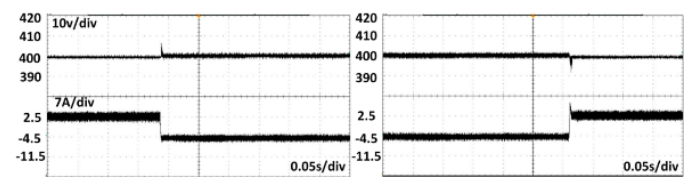

Fig.6-c the experiment waveform of Buck charge/discharge mode transition using FOPID controller

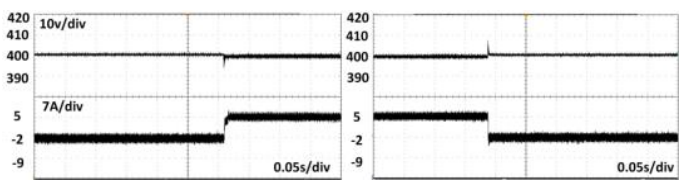

Fig.6-d the experiment waveform of Boost charge/discharge mode transition using FOPID controller

By using FOPID controller, the maximum voltage overshoot of mode transition is about $8 \mathrm{~V}$ in Buck and the regulation time is about $15 \mathrm{~ms}$. The maximum voltage overshoot is about $9 \mathrm{~V}$ in Boost

\section{References}

[1] Zhao Biao, in Al, Wang Liwen.Z source DC-DC converter and its control strategy [J].Chinese Journal of Electrical Engineering, 2011, 09:43-49.

[2] Cheng Hong, Gao Qiaomei, Zhu Jinbiao, Yang Xiaokang, Wang Cong.Dynamic modeling and minimum return power control of a bi-directional full bridge DC-DC converter based on dual phase shift control [J].Journal of Electrical Technology, 2014, 03:245-253.

[3] Li Hequn, GaoQiaomei, Yang Xiaokang, Cheng Hong. Research on single phase shift closed loop control of DC-DC converter with bi-directional full bridge [J].Mining automation, 2014, 04:63-67.

[4] Zhao Biao, in $\mathrm{Al}$, Sun Weixin. Double phase full bridge DC-DC converter and its power return characteristics analysis $[\mathrm{J}]$. Chinese Journal of Electrical Engineering. 2012, 12:43-50

[5] Lin Weiming, Guo Xiaojun, Huang Chao. Improved one cycle control strategy of bidirectional high ratio of switching DC-DC converter $[\mathrm{J}]$. Chinese Journal of Electrical Engineering. 2012, 21:31-37.

[6] Imamura,Y; Ramadan,H.A; SihunYang; Dousoky,G.M; Shoyama, M. Seamless Dynamic Model for DC-DC Converters Applicable to Bi-directional Power Transfer. Power Electronics and Applications (EPE), 2013 15th European Conference on.1-10.

[7] Ki-Man Kim, Sang-Hoon Park, Jung-HyoLee,etal.Mode Change Method of Bi-directional DC/DC Converter for Electric Vehicle[A].In Proceedings of the 8th International Conferenceon Power Electronics and ECCE Asia[C].Jeju,2011:2687-2693.

[8] Feng liuxin. Hybrid bi-directional converter mode transition control. Power electronic technology, 2014 (7):48-50)

[9] D. Maiti, A. Acharya M. Chakraborty, A. Konar, R. Janarthanan, "Tuning PID and Fractional PID Controllers using the Integral Time Absolute Error and the regulation time is about $20 \mathrm{~ms}$. Hardware experimental results are basically in accordance with the simulation results.

\section{CONCLUSION}

Based on the method of dual carrier modulation, the duty cycle of mode transition changes from discontinuous to continuous and the control method can also be simplified in H-bridge bi-directional DC-DC converter. By using PI controller, the overshoot voltage and time of regulation can be reduced, which protects the power tube and quickens the stability of DC bus voltage, effectively. But an FOPID can be more effective in dynamic performance of mode transition compared to a PI. The results of both simulation and experiment verify the feasibility and superiority of this method that offers some reference value for industrial application.

Criterion" 4th International Conference on Information and Automation for Sustainability, 2008, pp. 457-462.

[10] M. Zamani, M. Karimi-Ghartemani, N. Sadati, and M. Parniani, "Design of a fractional order PID controller for an AVR using particle swarm optimization, " Control Engineering Practice, vol. 17, pp.1380-1387, 2009.

[11] I.Pan and S. Das, "Frequency domain design of fractional order PID controller for AVR system using chaotic multi-objective optimization, "International Journal of Electrical Power \& Energy Systems, vol. 51, pp. 106118,2013

[12] Y. Tang, M. Cui, C.Hua, L. Li, and Y. Yang, "Potimum design of fractional order PID controller for AVR system using chaotic ant swarm, "Expert Systems with Applications, vol. 39, pp. 6887-6896, 2012.

[13] K. Arab Tehrani, A. Amirahmadi, S. M. R. Rafiei. "Design of fractional order PID controller for boost converter based on Multi-Objective optimization," Power Electronics and Motion Control Conference (EPE/PEMC), 2010 14th International, Ohrid, 2010, pp. T3-179-T3-185.

[14] D. Xue, C. Zhao, Y. Q. Chen, "Fractional Order PID Control of a DC Motor with Elastic Shaft: A Case Study", the 2006 American Control Conference, Minneapolis, Minnesota, USA, June 14-16, 2006,pp. 3182-3187.

[15] Cao Jun-yi, Cao Bing-gang."Digital realization and characteristics of fractional order controllers [J]".Control Theory \& Applications. 2006, 05:791$794+799$ 